\title{
Facile approach to prepare 3-cyanopyridin-2(1H)-one derivatives
}

\author{
Gagik Melikyan* and Alexander Piroyan \\ Department of Organic Chemistry, Yerevan State University, Armenia A.Manoogian 1, \\ 375025 Yerevan, Armenia \\ E-mail: suren@netsys.am
}

\begin{abstract}
Dedicated to Professor Armand Lattes on $50^{\text {th }}$ anniversary of his educational and scientific activity
\end{abstract}

\begin{abstract}
A new and efficient high yielding synthesis of N-substituted 3-cyanopyridin-2-ones has been developed. The reaction of ylidencyanoacetic acid ethyl esters and dimethylformamide dimethyl acetal has been investigated. Intermediate dimethylamino derivatives so formed were treated with various primary amines in xylene. The targeted 3-cyanopyridin-2-ones were obtained in good yields.
\end{abstract}

Keywords: 3-Cyanopyridin-2-ones, cardiotonic agent, milrinone, alkaloid, ricinine

\section{Introduction}

The pharmacological and physiological activity of 3-cyanopyridin-2-ones has attracted much attention in recent years with the synthesis and the study of the non-glycosidic cardiotonic agent milrinone, ${ }^{1,2}$ as well as with a number of 3-cyanopyridin-2-one derivatives which proved to be active against the herpes virus and the human immunodeficiency virus. ${ }^{3,4}$ The 3-cyanopyridin-2one nucleus is also the structural basis of the alkaloid ricinine - the first known alkaloid containing a cyano-group (Figure 1).<smiles>Cc1[nH]c(=O)c(C#N)cc1-c1ccncc1</smiles>

milrinone<smiles>COc1ccn(C)c(=O)c1C#N</smiles>

ricinine

\section{Figure 1}




\section{Results and Discussion}

There are many reported methods for the formation of 3-cyanopyridin-2-ones. The main strategy involves the condensation of $\beta$-enaminoderivatives of carbonyl compounds with cyanoacetic acid amides or malononitrile.,

We report herein a new, efficient and convenient synthetic approach to 3-cyanopyridin-2ones utilizing various ylidenecyanoacetic acid ethyl esters and dimethylformamide dimethyl acetal as starting materials. The reaction was carried out in anhydrous xylene under reflux for 3 hours. The intermediate dimethylamino derivatives 1-3 so formed were treated with primary amines in xylene under reflux for 13-15 hours to yield the targeted 3-cyanopyridin-2-ones 4-15 in good yields. The course of the reaction was monitored by the evolution of gaseous dimethylamine (Scheme 1).

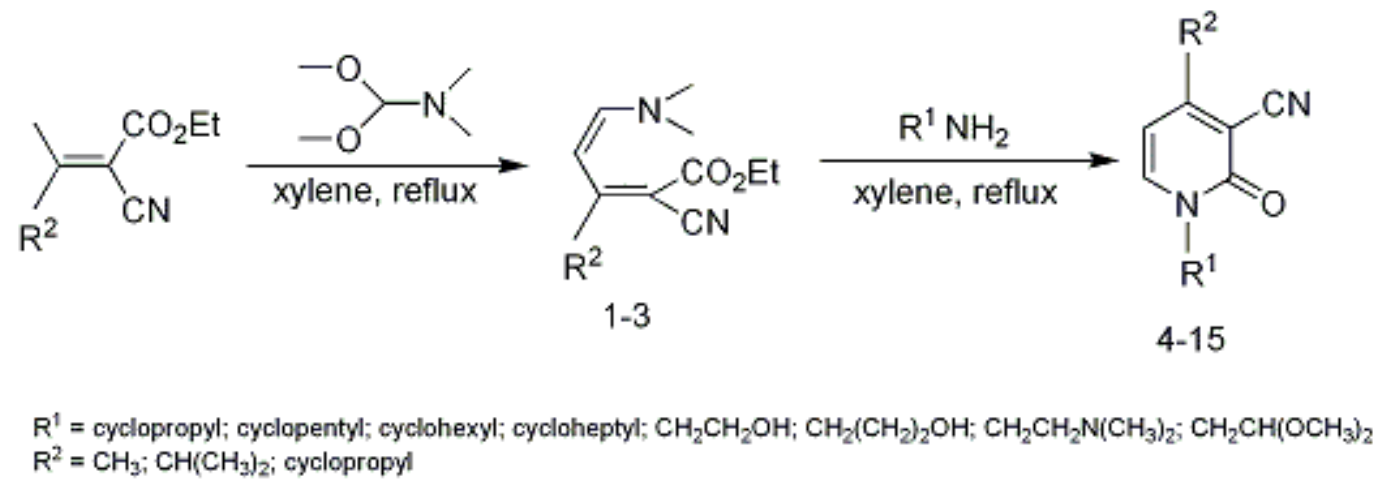

\section{Scheme 1}

It is known that bis lactams are the structural component of some natural compounds. ${ }^{7}$ Considering this fact we have prepared the corresponding bis-pyridinone 16 using ethylene diamine as the primary amine (Scheme 2).

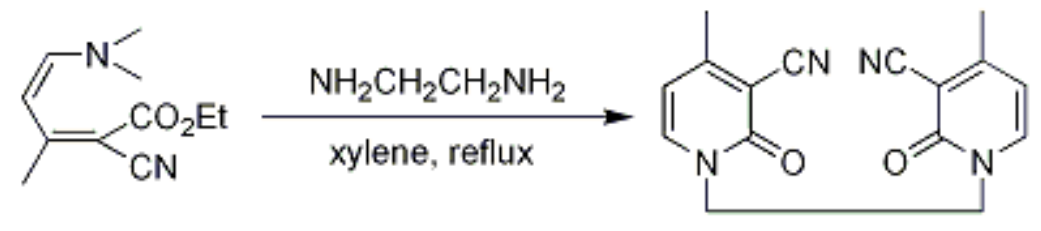

1

16

\section{Scheme 2}

Taking into account that pyridinones with functionalized substituents, in particular containing unsaturated bonds are an interesting class of compounds, we also carried out the condensation of 1-oxyethyl- and 1-oxypropyl-3-cyano-4-methylpyridin-2-ones with 
dimethylformamide dimethyl acetal in xylene under reflux. The targeted products 17, 18 were isolated in $70-75 \%$ yields (Scheme 3 ).

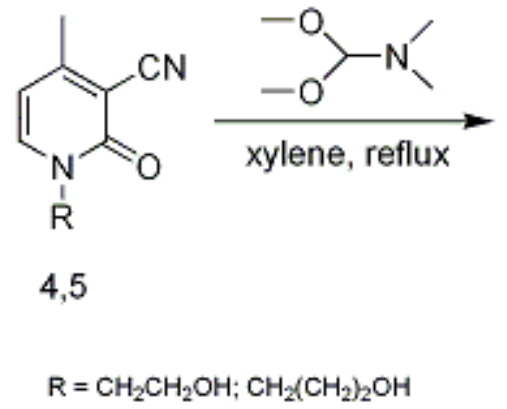<smiles>[R]n1ccc(/C=C/N(C)C)c(C#N)c1=O</smiles>

17,18

\section{Scheme 3}

In conclusion, we have developed a new and efficient high yielding synthesis of $\mathrm{N}$ substituted 3-cyanopyridin-2-ones.

\section{Experimental Section}

General Procedures. All melting points were determined on a Kofler hot-stage microscope and are uncorrected. ${ }^{1} \mathrm{H}-\mathrm{NMR}$ and ${ }^{13} \mathrm{C}-\mathrm{NMR}$ spectra were obtained on a "Varian Mercury 300" spectrometer at 300 and $75 \mathrm{MHz}$ with tetramethylsilane (TMS) as internal reference in DMSO$\mathrm{d}_{6}$, DMSO- $\mathrm{d}_{6}: \mathrm{CCl}_{4}(1: 3)$ and DMSO- $\mathrm{d}_{6}: \mathrm{CCl}_{4}(3: 1)$ solutions at $303 \mathrm{~K}$. IR spectra were recorded on a NEXUS FT-IR. Elemental analysis satisfactory obtained: $\mathrm{C} \pm 0.32, \mathrm{H} \pm 0.20, \mathrm{~N} \pm$ 0.27 .

\section{Compounds $1-3$ and 17, 18}

A solution of each initial compound $(0,01 \mathrm{~mol})$ in anhydrous xylene $(20 \mathrm{ml})$ was treated with dimethylformamide dimethyl acetal $(0,011 \mathrm{~mol})$. The reaction mixture was refluxed for 3 hours, then allowed to cool. Light petroleum $\left(10 \mathrm{ml}\right.$, b.p. $\left.60-80{ }^{\circ} \mathrm{C}\right)$ was added to the reaction mixture at room temperature. The solid products so formed were collected by filtration and crystallized from light petroleum. Data of the compounds are shown below.

Ethyl 2-cyano-5-(dimethylamino)-3-methylpenta-2,4-dienoate (1). Yellow crystals, yield $55 \%$, m.p. $98-101^{\circ} \mathrm{C}$; mixture of two stereoisomers : A and B.

A $(\sim 66 \%)$ :

${ }^{1} \mathrm{H}-\mathrm{NMR}\left(300 \mathrm{MHz}, \mathrm{DMSO}-\mathrm{d}_{6}\right) \delta 1.30(\mathrm{t}, 3 \mathrm{H}, \mathrm{J}=7.1 \mathrm{~Hz}), 2.25(\mathrm{~s}, 3 \mathrm{H}), 3.00(\mathrm{~s}, 3 \mathrm{H}), 3.22(\mathrm{~s}, 3 \mathrm{H})$, $4.12(\mathrm{q}, 2 \mathrm{H}, J=7.1 \mathrm{~Hz}), 6.99(\mathrm{~d}, 1 \mathrm{H}, J=13.1 \mathrm{~Hz}), 7.58$ (d, 1H, $J=13.1 \mathrm{~Hz})$.

$\mathrm{B}(\sim 34 \%)$ :

${ }^{1} \mathrm{H}-\mathrm{NMR}\left(300 \mathrm{MHz}, \mathrm{DMSO}-\mathrm{d}_{6}\right) \delta 1.29(\mathrm{t}, 3 \mathrm{H}, J=7.1 \mathrm{~Hz}), 2.41(\mathrm{~s}, 3 \mathrm{H}), 3.00(\mathrm{~s}, 3 \mathrm{H}), 3.22(\mathrm{~s}, 3 \mathrm{H})$, $4.11(\mathrm{q}, 2 \mathrm{H}, J=7.1 \mathrm{~Hz}), 5.60(\mathrm{~d}, 1 \mathrm{H}, J=12.7 \mathrm{~Hz}), 7.58(\mathrm{~d}, 1 \mathrm{H}, J=12.7 \mathrm{~Hz})$. 
Ethyl 2-cyano-5-(dimethylamino)-3-isopropylpenta-2,4-dienoate (2). Yellow crystals, yield $56 \%$, m.p. $128-130^{\circ} \mathrm{C}$; ${ }^{1} \mathrm{H}-\mathrm{NMR}\left(300 \mathrm{MHz}, \mathrm{DMSO}-\mathrm{d}_{6}\right) \delta 1.31(\mathrm{t}, 3 \mathrm{H}, J=7.1 \mathrm{~Hz}), 1.36(\mathrm{~d}, 6 \mathrm{H}$, $J=7.2 \mathrm{~Hz}$ ), 3.10 (s, 6H), 3.30 (sept., $1 \mathrm{H}, J=7.2 \mathrm{~Hz}), 4.12$ (q, 2H, J=7.1Hz), 6.92 (d, 1H, $J=13.3 \mathrm{~Hz}), 7.69(\mathrm{~d}, 1 \mathrm{H}, J=13.3 \mathrm{~Hz})$;

Ethyl 2-cyano-3-cyclopropyl-5-(dimethylamino)penta-2,4-dienoate (3). Dark-yellow crystals, yield 59\%, m.p. 93-95 C; ${ }^{1} \mathrm{H}-\mathrm{NMR}\left(300 \mathrm{MHz}, \mathrm{DMSO}-\mathrm{d}_{6}\right) \delta 0.69$ (q, 2H, J=5.8Hz), 1.07 (m, 2H), $1.30(\mathrm{t}, 3 \mathrm{H}, J=7.1 \mathrm{~Hz}), 1.69(\mathrm{~m}, 1 \mathrm{H}), 3.01(\mathrm{~s}, 3 \mathrm{H}), 3.22(\mathrm{~s}, 3 \mathrm{H}), 4.12$ (q, 2H, J=7.1Hz), 6.87 (d, $1 \mathrm{H}, J=13.0 \mathrm{~Hz}), 7.86(\mathrm{~d}, 1 \mathrm{H}, J=12.9 \mathrm{~Hz})$;

3-Cyano-4-[2-(dimethylamino)vinyl]-1-(2-hydroxyethyl)pyridin-2(1H)-one (17): Green crystals, yield 80\%, m.p. 170-172 ${ }^{\circ} \mathrm{C} ;{ }^{1} \mathrm{H}-\mathrm{NMR}\left(300 \mathrm{MHz}, \mathrm{DMSO}-\mathrm{d}_{6}\right) \delta 3.06(\mathrm{~s}, 6 \mathrm{H}), 3.62(\mathrm{t}$, $2 \mathrm{H}, J=5.2 \mathrm{~Hz}), 3.83$ (t, $2 \mathrm{H}, J=5.3 \mathrm{~Hz}), 4.61$ (br., $1 \mathrm{H}), 5.15(\mathrm{~d}, 1 \mathrm{H}, J=13.1 \mathrm{~Hz}), 6.34(\mathrm{~d}, 1 \mathrm{H}$, $J=7.5 \mathrm{~Hz}), 7.33(\mathrm{~d}, 1 \mathrm{H}, J=7.5 \mathrm{~Hz}), 7.58(\mathrm{~d}, 1 \mathrm{H}, J=13.1 \mathrm{~Hz})$;

3-Cyano-4-[2-(dimethylamino)vinyl]-1-(3-hydroxypropyl)pyridin-2(1H)-one (18). Darkgreen crystals, yield 76\%, m.p. $165-167{ }^{\circ} \mathrm{C} ;{ }^{1} \mathrm{H}-\mathrm{NMR}\left(300 \mathrm{MHz}, \mathrm{DMSO}-\mathrm{d}_{6}\right) \delta 1.78(\mathrm{p}, 2 \mathrm{H}$, $J=6.4 \mathrm{~Hz}$ ), 3.06 (s, 6H), 3.42 (br. t, 2H, $J=5.7 \mathrm{~Hz}$ ), 3.86 (t, 2H, $J=6.7 \mathrm{~Hz}$ ), 4.28 (br., 1H), 5.15 (d, $1 \mathrm{H}, J=13.1 \mathrm{~Hz}), 6.38$ (d, 1H, J=7.5Hz), 7.36 (d, 1H, $J=7.5 \mathrm{~Hz}), 7.59$ (d, 1H, $J=13.1 \mathrm{~Hz})$;

\section{Compounds 4-16}

A mixture of each dimethylamino derivatives (1-3) (0,005 mol) and the corresponding amine $(0,015 \mathrm{~mol})$ (in case of ethylenediamine - 0,0075 mol) in xylene $(10 \mathrm{ml})$ was refluxed for $13-15$ hours. The progress of the reaction was monitored by the evolution of gaseous dimethylamine. After completion of the reaction, the mixture was cooled to room temperature. Then, light petroleum $\left(10 \mathrm{ml}\right.$, b.p. $\left.60-80{ }^{\circ} \mathrm{C}\right)$ was added to the reaction mixture. The solid products were filtered off and washed with light petroleum $(20 \mathrm{ml})$ and the crude products isolated. The crude products were purified by recrystallization from xylene. Data of the compounds are shown below:

3-Cyano-1-(2-hydroxyethyl)-4-methylpyridin-2(1H)-one (4). Beige crystals, yield 55\%, m.p. 128-130 ${ }^{\circ} \mathrm{C}$; IR (vaseline, $\mathrm{cm}^{-1}$ ) 3412, 2223, 1649, 1598; ${ }^{1} \mathrm{H}-\mathrm{NMR}$ (300 MHz, DMSO-d 6 ) $\delta 2.40$ (s, 3H), 3.66 (t, 2H, J=5.2Hz), 3.98 (t, 2H, J=5.2Hz), 4.64 (br., 1H), 6.19 (d, 1H, J=6.9Hz), 7.74 $(\mathrm{d}, 1 \mathrm{H}, J=6.9 \mathrm{~Hz}) ;{ }^{13} \mathrm{C}-\mathrm{NMR}\left(75 \mathrm{MHz}, \mathrm{DMSO}-\mathrm{d}_{6}: \mathrm{CCl}_{4} / 1: 3\right) \delta 20.26,51.55,58.15,102.66$, $106.38,114.79,143.13,158.51,158.95$.

3-Cyano-1-(3-hydroxypropyl)-4-methylpyridin-2(1H)-one (5). White crystals, yield 60\%, m.p. 136-138 ${ }^{\circ} \mathrm{C}$; IR (vaseline, $\mathrm{cm}^{-1}$ ) 3410, 2225, 1651, 1601; ${ }^{1} \mathrm{H}-\mathrm{NMR}$ (300 MHz, DMSO-d 6 ) $\delta$ 1.83 ( p, 2H, $J=6.4 \mathrm{~Hz}), 2.40(\mathrm{~s}, 3 \mathrm{H}), 3.44$ (q, 2H, $J=5.4 \mathrm{~Hz}), 4.01$ (t, 2H, J=6.9Hz), 4.32 (br. t, $1 \mathrm{H}, J=5.0 \mathrm{~Hz}), 6.21(\mathrm{~d}, 1 \mathrm{H}, J=6.9 \mathrm{~Hz}), 7.81(\mathrm{~d}, 1 \mathrm{H}, J=6.8 \mathrm{~Hz}) ;{ }^{13} \mathrm{C}-\mathrm{NMR}\left(75 \mathrm{MHz}, \mathrm{DMSO}-\mathrm{d}_{6}\right.$ : $\left.\mathrm{CCl}_{4} / 1: 3\right) \delta 20.22,30.92,46.51,57.01,102.97,106.88,114.66,142.36,158.39,158.90$.

3-cyano-1-cyclopropyl-4-methylpyridin-2(1H)-one (6). White crystals, yield 52\%, m.p. 93-94 ${ }^{\circ} \mathrm{C} ;{ }^{1} \mathrm{H}-\mathrm{NMR}\left(300 \mathrm{MHz}, \mathrm{DMSO}-\mathrm{d}_{6}\right) \delta 0.89(\mathrm{~m}, 2 \mathrm{H}), 1.08(\mathrm{~m}, 2 \mathrm{H}), 2.25(\mathrm{~s}, 1 \mathrm{H}), 3.34(\mathrm{~m}, 1 \mathrm{H})$, $6.17(\mathrm{~d}, 1 \mathrm{H}, J=7.1 \mathrm{~Hz}), 7.65$ (d, 1H, $J=7.1 \mathrm{~Hz})$.

3-Cyano-1-cyclopentyl-4-methylpyridin-2(1H)-one (7). Beige crystals, yield 57\%, m.p. 133$134{ }^{\circ} \mathrm{C}$; IR (vaseline, $\mathrm{cm}^{-1}$ ) 2226, 1651, 1600; ${ }^{1} \mathrm{H}-\mathrm{NMR}\left(300 \mathrm{MHz}, \mathrm{DMSO}-\mathrm{d}_{6}\right) \delta 1.63-2.19(\mathrm{~m}$, 
$8 \mathrm{H}), 2.40(\mathrm{~s}, 3 \mathrm{H}), 5.06(\mathrm{p}, 1 \mathrm{H}, J=7.9 \mathrm{~Hz}), 6.23(\mathrm{~d}, 1 \mathrm{H}, J=7.1 \mathrm{~Hz}), 7.76(\mathrm{~d}, 1 \mathrm{H}, J=7.1 \mathrm{~Hz}) ;{ }^{13} \mathrm{C}-$ NMR (75 MHz, DMSO-d $6: \mathrm{CCl}_{4} /$ 1:3) $\delta$ 20.09, 23.63, 31.08, 56.82, 102.85, 107.37, 114.66, 138.42, 157.54, 158.92.

3-Cyano-1-cyclohexyl-4-methylpyridin-2(1H)-one (8). White needles, yield 62\%, m.p. 136$138{ }^{\circ} \mathrm{C}$; ${ }^{1} \mathrm{H}-\mathrm{NMR}\left(300 \mathrm{MHz}, \mathrm{DMSO}_{6}\right) \delta 1.17-1.98(\mathrm{~m}, 10 \mathrm{H}), 2.39(\mathrm{~s}, 3 \mathrm{H}), 4.65(\mathrm{tt}, 1 \mathrm{H}$, $J=3.6 \mathrm{~Hz}, J=11.7 \mathrm{~Hz}), 6.23$ (d, 1H, $J=7.1 \mathrm{~Hz}), 7.78$ (d, $1 \mathrm{H}, J=7.1 \mathrm{~Hz})$;

3-Cyano-1-cycloheptyl-4-methylpyridin-2(1H)-one (9). White crystals, yield 60\%, m.p. 111$113{ }^{\circ} \mathrm{C} ;{ }^{1} \mathrm{H}-\mathrm{NMR}\left(300 \mathrm{MHz}, \mathrm{DMSO}_{6}\right) \delta 1.54-1.91(\mathrm{~m}, 12 \mathrm{H}), 2.39(\mathrm{~s}, 3 \mathrm{H}), 4.70-4.83(\mathrm{~m}, 1 \mathrm{H})$, $6.23(\mathrm{~d}, 1 \mathrm{H}, J=7.1 \mathrm{~Hz}), 7.76(\mathrm{~d}, 1 \mathrm{H}, J=7.1 \mathrm{~Hz})$;

3-Cyano-1-cyclopentyl-4-isopropylpyridin-2(1H)-one (10). Grey crystals, yield 55\%, m.p. 119-120 ${ }^{\circ} \mathrm{C}$; IR (vaseline, $\mathrm{cm}^{-1}$ ) 2231, 1649, 1600; ${ }^{1} \mathrm{H}-\mathrm{NMR}\left(300 \mathrm{MHz}, \mathrm{DMSO}-\mathrm{d}_{6}\right) \delta 1.27$ (d, $6 \mathrm{H}, J=6.9 \mathrm{~Hz}), 1.63-2.18(\mathrm{~m}, 8 \mathrm{H}), 3.17$ ( sept., $1 \mathrm{H}, J=6.8 \mathrm{~Hz}), 5.06$ ( p, 1H, J=7.9Hz), 6.27 (d, $1 \mathrm{H}, J=7.3 \mathrm{~Hz}), 7.82(\mathrm{~d}, 1 \mathrm{H}, J=7.3 \mathrm{~Hz}) ;{ }^{13} \mathrm{C}-\mathrm{NMR}\left(75 \mathrm{MHz}, \mathrm{DMSO}-\mathrm{d}_{6}: \mathrm{CCl}_{4} / 1: 3\right) \delta 21.11$, 23.62, 31.01, 32.16, 56.90, 101.27, 102.76, 114.36, 139.27, 159.10, 166.72.

3-Cyano-1-cyclohexyl-4-isopropylpyridin-2(1H)-one (11). Beige crystals, yield 68\%, m.p. 171-172 ${ }^{\circ} \mathrm{C} ;{ }^{1} \mathrm{H}-\mathrm{NMR}\left(300 \mathrm{MHz}, \mathrm{DMSO}_{\mathrm{d}}\right.$ ) $\delta 1.27$ (d, 6H, J=6.8Hz), 1.44-1.94 (m, 10H), 3.17 ( sept., $1 \mathrm{H}, J=6.7 \mathrm{~Hz}), 4.66$ (tt, $1 \mathrm{H}, J=3.6 \mathrm{~Hz}, J=11.5 \mathrm{~Hz}), 6.27(\mathrm{~d}, 1 \mathrm{H}, J=7.2 \mathrm{~Hz}) \mathrm{ppm} 7.83(\mathrm{~d}, 1 \mathrm{H}$, $J=7.2 \mathrm{~Hz}$;

3-Cyano-4-cyclopropyl-1-[2-(dimethylamino)ethyl]pyridin-2(1H)-one (12). White crystals, yield 55\%, m.p. $71-74{ }^{\circ} \mathrm{C}$; IR (vaseline, $\mathrm{cm}^{-1}$ ) 2235, 1653, 1599; ${ }^{1} \mathrm{H}-\mathrm{NMR}$ (300 MHz, DMSO-d 6 ) $\delta 0.97(\mathrm{~m}, 2 \mathrm{H}), 1.26(\mathrm{~m}, 2 \mathrm{H}), 2.17(\mathrm{td}, 1 \mathrm{H}, J=4.7 \mathrm{~Hz}, J=8.8 \mathrm{~Hz}), 2.22(\mathrm{~s}, 6 \mathrm{H}), 2.52(\mathrm{t}, 2 \mathrm{H}$, $J=6.2 \mathrm{~Hz}), 3.95(\mathrm{t}, 2 \mathrm{H}, J=6.1 \mathrm{~Hz}), 5.65(\mathrm{~d}, 1 \mathrm{H}, J=7.2 \mathrm{~Hz}), 7.67(\mathrm{~d}, 1 \mathrm{H}, J=7.2 \mathrm{~Hz}) ;{ }^{13} \mathrm{C}-\mathrm{NMR}(75$ MHz, DMSO-d $\left.{ }_{6}: \mathrm{CCl}_{4} / 1: 3\right) \delta 10.36,14.21,44.89,46.06,56.87,99.09,101.66,115.00,142.85$, $158.55,164.44$.

3-Cyano-4-cyclopropyl-1-(2,2-dimethoxyethyl)pyridin-2(1H)-one (13). White crystals, yield 54\%, m.p. 86-87 ${ }^{\circ} \mathrm{C}$; IR (vaseline, $\mathrm{cm}^{-1}$ ) 2230, 1649, 1600; ${ }^{1} \mathrm{H}-\mathrm{NMR}$ (300 MHz, DMSO-d 6 ) $\delta$ $0.99(\mathrm{~m}, 2 \mathrm{H}), 1.28(\mathrm{~m}, 2 \mathrm{H}), 2.19(\mathrm{~m}, 1 \mathrm{H}), 3.36(\mathrm{~s}, 6 \mathrm{H}), 3.95(\mathrm{~d}, 2 \mathrm{H}, J=5.3 \mathrm{~Hz}), 4.55(\mathrm{t}, 1 \mathrm{H}$, $J=5.3 \mathrm{~Hz}), 5.68(\mathrm{~d}, 1 \mathrm{H}, J=7.3 \mathrm{~Hz}), 7.64(\mathrm{~d}, 1 \mathrm{H}, J=7.3 \mathrm{~Hz}) ;{ }^{13} \mathrm{C}-\mathrm{NMR}\left(75 \mathrm{MHz}, \mathrm{DMSO}-\mathrm{d}_{6}: \mathrm{CCl}_{4} /\right.$ $1: 3) \delta 10.52,14.33,50.10,53.97,99.42,100.69,101.62,114.82,143.31,158.70,165.02$.

3-Cyano-1,4-dicyclopropylpyridin-2(1H)-one (14). White crystals, yield 56\%, m.p. 154-156 ${ }^{\circ} \mathrm{C}$; IR (vaseline, $\mathrm{cm}^{-1}$ ) 2222, 1655, 1604; ${ }^{1} \mathrm{H}-\mathrm{NMR}\left(300 \mathrm{MHz}, \mathrm{DMSO}-\mathrm{d}_{6}\right) \delta 0.86(\mathrm{~m}, 2 \mathrm{H}), 0.95$ $(\mathrm{td}, 2 \mathrm{H}, J=4.6 \mathrm{~Hz}, J=7.2 \mathrm{~Hz}), 1.07(\mathrm{~m}, 2 \mathrm{H}), 1.26(\mathrm{~m}, 2 \mathrm{H}), 2.18(\mathrm{tt}, 1 \mathrm{H}, J=4.8 \mathrm{~Hz}, J=8.2 \mathrm{~Hz}), 3.31$ $(\mathrm{tt}, 1 \mathrm{H}, J=4.2 \mathrm{~Hz}, J=7.5 \mathrm{~Hz}), 5.65(\mathrm{~d}, 1 \mathrm{H}, J=7.4 \mathrm{~Hz}), 7.58(\mathrm{~d}, 1 \mathrm{H}, J=7.3 \mathrm{~Hz}) ;{ }^{13} \mathrm{C}-\mathrm{NMR}(75 \mathrm{MHz}$, DMSO-d $\left.{ }_{6}: \mathrm{CCl}_{4} / 1: 3\right) \delta 6.07,10.35,14.20,31.65,99.46,101.70,114.92,141.10,159.65$, 164.21 .

3-Cyano-1-cyclohexyl-4-cyclopropylpyridin-2(1H)-one (15). Grey crystals, yield 71\%, m.p. 176-178 ${ }^{\circ} \mathrm{C} ;{ }^{1} \mathrm{H}-\mathrm{NMR}\left(300 \mathrm{MHz}, \mathrm{DMSO}-\mathrm{d}_{6}\right) \delta 0.96(\mathrm{~m}, 2 \mathrm{H}), 1.26(\mathrm{td}, 2 \mathrm{H}, J=4.6 \mathrm{~Hz}, J=6.9 \mathrm{~Hz})$, $1.40-1.95$ (m, 10H), $2.18(\mathrm{tt}, 1 \mathrm{H}, J=4.8 \mathrm{~Hz}, J=8.3 \mathrm{~Hz}), 4.63(\mathrm{tt}, 1 \mathrm{H}, J=3.3 \mathrm{~Hz}, J=11.7 \mathrm{~Hz}), 5.71$ (d, $1 \mathrm{H}, J=7.4 \mathrm{~Hz}), 7.70(\mathrm{~d}, 1 \mathrm{H}, J=7.4 \mathrm{~Hz})$; 


\section{3-Cyano-1-[2-(3-cyano-4-methyl-2-oxopyridin-1(2H)-yl)ethyl]-4-methylpyridin-2(1H)-one}

(16). Beige crystals, yield 78\%, m.p. $>250{ }^{\circ} \mathrm{C}$; IR (vaseline, $\mathrm{cm}^{-1}$ ) 2235, 1650, 1600; ${ }^{1} \mathrm{H}-\mathrm{NMR}$ $\left(300 \mathrm{MHz}, \mathrm{DMSO}-\mathrm{d}_{6}\right) \delta 2.35(\mathrm{~s}, 6 \mathrm{H}), 4.23(\mathrm{~s}, 4 \mathrm{H}), 6.29$ (d, 2H, J=6.9Hz), 7.72 (d, 2H, J=6.9Hz); ${ }^{13} \mathrm{C}-\mathrm{NMR}\left(75 \mathrm{MHz}, \mathrm{DMSO}-\mathrm{d}_{6}: \mathrm{CCl}_{4} /\right.$ 3:1) $\delta$ 20.47, 47.69, 102.40, 107.71, 115.27, 142.69, $159.32,159.95$.

\section{References}

1. Shiae, M.-J.; Shyu, L.-M.; Chen, C.-F. Heterocycles 1990, 31, 523.

2. Hopkins, S. J. Drugs of Today 1990, 26, 295.

3. Saad, H. A.; Mokbil, M. N.; El-Gendy, A. M.; Haikal, A. Z. Synthetic Communications 2002, 32, 1189.

4. Dolle, V.; Fan, E.; Nguyen, C. H.; Bisagni, E. J. Med. Chem. 1995, 38, 4679.

5. Litvinov, V. P. Phosphoros, Sulfur, Silicon and Relat. Elem. 1993, 74, 139.

6. Al-Omran, F.; Al-Awadhi, N.; Abdel, K. M. M.; Kaul, K.; El-Khair, A. A.; Elnagdi, M. H. J.Chem. Res. (S) 1997, 3, 84.

7. Wasserman, H. H.; DeSimone, R. J. Am. Chem. Soc. 1993, 115, 8457. 